\title{
Polyploidy and Genome Evolution in Plants
}

\section{Pamela S. Soltis',2, D. Blaine Marchant ${ }^{1,3}$, Yves Van de Peer ${ }^{4}$, and Douglas E. Soltis ${ }^{1,2,3}$}

1Florida Museum of Natural History, University of Florida, Gainesville, FL 32611, USA

${ }^{2}$ Genetics Institute, University of Florida, Gainesville, FL 32610, USA

${ }^{3}$ Department of Biology, University of Florida, Gainesville, FL 32611, USA

${ }^{4}$ Department of Plant Systems Biology, VIB (Flanders Institute of Biotechnology), B-9052

Ghent, Belgium, and the Bioinformatics and Evolutionary Genomics Research Group,

Department of Plant Biotechnology and Genetics, Ghent University, B-9052 Ghent, Belgium 


\begin{abstract}
Plant genomes vary in size and complexity, fueled in part by processes of whole-genome duplication (WGD; polyploidy) and subsequent genome evolution. Despite repeated episodes of WGD throughout the evolutionary history of angiosperms in particular, the genomes are not uniformly large, and even plants with very small genomes carry the signatures of ancient duplication events. The processes governing the evolution of plant genomes following these ancient events are largely unknown. Here, we consider mechanisms of diploidization, evidence of genome reorganization in recently formed polyploid species, and macroevolutionary patterns of WGD in plant genomes and propose that the ongoing genomic changes observed in recent polyploids may illustrate the diploidization processes that result in ancient signatures of WGD over geological timescales.
\end{abstract}

\title{
Introduction
}

Plant genomes vary spectacularly in size, ranging from $0.063 \mathrm{~Gb}$ to $148.8 \mathrm{~Gb}$, a 2400 -fold difference $(1 ; \underline{w w w . d a t a . k e w . o r g / c v a l u e s})$. Much of this diversity results from differential expansion and loss of repeats (reviewed in 2), but an additional major driver shaping variation in genome size in plants is whole-genome duplication (WGD; also known as polyploidy, see Box for glossary of terms). Moreover, genome structure and gene content in plants are intimately tied to the history of WGD. We therefore contend that any understanding of plant genome structure, content, and evolution requires consideration of WGD and its consequences. 


\section{Polyploidy in Plants}

Polyploidy has long been considered an important mechanism of speciation in plants, particularly angiosperms (e.g., see reviews by 3-10). The frequency of polyploidy has typically been based on chromosome numbers, and estimates for major clades of green plants (Viridiplantae) have varied dramatically, ranging from very low in bryophytes to as high as $95 \%$ for ferns (6). For angiosperms (flowering plants) alone, estimates have ranged from $30-35 \%$ (4) to 70\% (11), with most estimates near 50\% (e.g., 5,12-14). Genomic data for plants have demonstrated a dazzling history of repeated WGDs throughout evolutionary history. Even the small genome of Arabidopsis thaliana (0.157 Gb) - this small size was one of the keys to the choice of this species as a genomic model - shows signatures of ancient WGD $(15,16)$. All angiosperms share an ancient WGD, as do all seed plants (17). Thus, in recent years, interpretations of plants as 'diploids' or 'polyploids' have been blurred, requiring much more nuanced vocabulary to describe plant genomes.

The origin of new species via polyploidy requires a series of seemingly low-probability events, including hybridization, unreduced gamete formation, establishment, and survival (see e.g., 18-20). Despite these apparent barriers, polyploid species are common in all floras worldwide and are particularly abundant at high latitudes and high elevations (e.g., 21, 22). The 'success' of polyploids is often attributed to the increased genetic diversity held within single polyploid individuals relative to that of their diploid progenitors (e.g., 7-9, 22-25). Moreover, this genetic diversity may be manifested in novelty at the biochemical, physiological, morphological, and ecological levels, giving polyploids an advantage, at least in the short term, over their diploid parents (e.g., 26, 27). The proximal reason behind 
polyploid success, therefore, may vary among species. However, if polyploidy per se were always a successful strategy, then plant genomes, such as that of $A$. thaliana, should show more obvious evidence of WGDs, such as high chromosome numbers, large genomes, and routinely duplicate (triplicate, quadruplicate, etc.) gene copies. Although many apparently recently formed polyploids exhibit these expected attributes of WGD, those species, such as A. thaliana, that are the products of ancient WGD harbor signatures of WGD within what are generally 'diploid' genomes, based on chromosome number, genome size, and gene copy number. Certainly, processes of diploidization are at play, leading to repeated cycles of polyploidy followed by diploidization followed by polyploidy, etc. (e.g., 28-30).

But what are these processes of diploidization, and how can they be reconciled with observations of gene family diversity, ancient signatures of WGD, and macroevolutionary patterns of polyploidization? In this paper, we will attempt to unify (i) hypothesized mechanisms of diploidization, (ii) data on genome reorganization shortly after polyploidization based on the evolutionary model, Tragopogon, and other recently formed polyploid species, and (iii) macroevolutionary patterns of WGD in plant genomes. We propose that the ongoing genomic changes observed in recent polyploids, such as Tragopogon, may illustrate the diploidization processes that result in ancient signatures of WGD over geological timescales.

\section{Mechanisms of Diploidization}

Repetitive DNA sequences comprise substantial portions of plant genomes (e.g. A. thaliana: 15-20\%; maize 85\%) and can largely influence genome size (e.g., 31, 32). Consequently, 
the two mechanisms by which these repetitive sequences (i.e. retrotransposons, DNA transposons, simple repeats) are lost (illegitimate recombination and unequal intra-strand homologous recombination) are the principal processes responsible for genome downsizing to a "diploid" state. Illegitimate recombination is hypothesized to remove DNA sequences via double-stranded breaks and/or slippage during replication, whereas unequal homologous recombination occurs between two repeat sequences and results in the loss of the DNA between the repeats, as well as one of the repeats (33). The relative importance of the two primary diploidization mechanisms appears to be species-specific in angiosperms, as the efficiency of DNA loss via either mechanism is highly variable and not phylogenetically related (33-35). Very few studies have addressed these processes outside of flowering plants; however, recent genomic sequencing of three conifer taxa has suggested that the enormous genomes of these taxa could be due to a very low recombination rate and high homologous chromosome fidelity, removing the opportunity for genome downsizing (36), at least via these mechanisms.

Although great strides have been made in determining the processes by which DNA content is reduced following polyploidy, few studies have addressed the second aspect of diploidization: the mechanisms by which entire chromosomes are lost. Only recently have studies of synthetic polyploids demonstrated the high prevalence of chromosomal instability immediately after genome duplication (37-40). While aneuploidy has been found to negatively correlate with fertility in synthetic Brassica napus polyploids (38) and pollen viability in synthetic wheat polyploids (40), the fact that chromosome number is no longer a suitable corollary for polyploidy history (e.g., A. thaliana has five chromosomes and five 
known polyploidy events), there must be some selective force to reduce chromosome number. Polyploid systems in which multiple temporal polyploid samplings (synthetic, nascent, ancient) occur will be critical for evaluating these diploidization processes in the future.

Diploidization also includes the loss of one homeologous copy of a duplicate gene pair, a process sometimes referred to as fractionation. Both genes and regulatory elements may be lost, with losses accumulating through time so that an ancient polyploid may have only a small number of genes retained in duplicate. These losses may be random with respect to parental genome, or losses may come from predominantly one parental genome, a process referred to as biased fractionation, the long-term result of which is a genome that resembles that of the alternative parent. Some genes are consistently returned to singleton status whereas others, such as transcription factors, are typically retained in duplicate (see below).

\section{Genome Reorganization in Recent Polyploids: Generating Novelty}

The genomes of newly formed natural polyploids, as well as those of synthetic polyploids, may experience rapid homeolog loss, as well as genome restructuring postpolyploidization, and altered patterns of gene expression may set the stage for subsequent loss of duplicate gene copies (e.g., 30, 38, 41-48). Both the extent and speed with which these diverse changes occur may vary considerably across diverse polyploid systems (reviewed in 30). 
In the recent and repeatedly formed allotetraploids Tragopogon mirus and T. miscellus, species in the sunflower family that originated in the early $20^{\text {th }}$ century (49), frequent homeolog loss, subfunctionalization, and major chromosomal changes, including translocations and compensated and non-compensated aneuploidy, were detected in natural populations, as well as in synthetic lines. Transcriptomic shock was observed; hybridization and polyploidy per se both play important roles in these young polyploids (50-54; reviewed in 43). Investigations of an older allotetraploid (T. castellanus) and its parents indicate that gene loss/expression changes and chromosomal alterations mirror what is seen in the recently formed T. mirus and T. miscellus and demonstrate that some of the alterations that occur immediately post-polyploidization may be retained over long evolutionary timeframes (55; Soltis, Soltis, Barbazuk, Schable et al. unpubl.). In Senecio cambrensis (also in the sunflower family and estimated to have originated in the 1700s; see review by Hegarty (42)), transcriptome shock was also detected; hybridization altered gene expression and DNA methylation, and genome duplication resulted in an additional burst of transcriptional and epigenetic change (42). In the grass Spartina anglica, which originated in the 1800s (see review by Ainouche (41)), rapid changes in gene expression were observed; hybridization played a larger role in methylation changes than polyploidy per se (41). Transcriptomic shock was detected; at the transcriptomic level, both hybridization and polyploidy are important. No chromosomal changes were noted.

Synthetic lines of older, established polyploids, including A. suecica $(44,45,56,57)$, Brassica napus $(38,46)$, and a synthetic Brassica hexaploid (47), have also been used to assess genomic and expression changes that arise shortly after polyploid formation. 
Transcriptome shock and rapid changes in expression and methylation are also observed, and the relative importance of hybridization and genome doubling seems to vary among species. The extent of homeolog loss versus expression changes also varies. Homeolog loss and chromosomal changes are frequent in Brassica (as in the natural polyploids of Tragopogon), but expression changes predominate in other systems (e.g., Arabidopsis, as in the natural polyploid, Spartina anglica, which has a stable karyotype).

Comparisons of synthetic and natural polyploids also indicate variation in the repeatability of evolution across independently formed polyploid lines, whether natural or synthetic. In Tragopogon, Senecio, and A. suecica, the consequences of polyploidy are repeated-that is, the evolutionary tape of life is replayed. However, in Brassica, a synthetic Arabidopsis hexaploid (47), and Spartina polyploids, independent origins respond differently. However, many aspects of polyploidy cannot be compared across all of these systems because of large gaps in the overall data set. For example, other features of polyploid genomes that may contribute to both genomic novelty in the short term and lead to genome downsizing over the longer term - e.g., transposon activity, methylation, subfunctionalization, and proteomic diversity - are only available for a few systems (30).

\section{Macroevolutionary Patterns of Genome Evolution in Plants}

The fact that there are many recognizable polyploids of fairly recent origin, but relatively little evidence for many ancient WGD events (paleopolyploidy; at least within the same evolutionary lineage), provides an interesting paradox. Although methods and data for detecting ancient WGDs are still limited, the inferred number of such events is increasing 
rapidly; tetraploid cottons $(2 n=52)$, for example, originated in the last one million years (58) and have an estimated 30-36-fold duplication of ancestral angiosperm genes (59). However, even as the picture of ancient WGDs is clarified, the number of such events will likely continue to underestimate the frequency of extant polyploids. This relative paucity of paleopolyploidy may reflect undetected WGDs due to limited genomic data; however, if real, this low frequency of ancient WGD suggests that polyploidy may be an evolutionary dead end, except perhaps in specific cases. Indeed, at some time in evolution, organisms that underwent and survived WGDs must have had an adaptive advantage. Examples of ancient WGD events that have been established on the longer term are one or two WGDs early in the evolution of seed and flowering plants (17), one WGD that is ancestral to most or all of the eudicots $(60,61)$, and one or two that occurred early in the monocot lineage $(16$, 59). Therefore, a question that has received much attention of late is whether these key ancient WGDs, which in many cases characterize major lineages of flowering plants, have survived by coincidence, or whether they may have originated in concert, at very specific geological times, for instance during times of major ecological or environmental upheaval, and/or periods of extinction. In this respect, one of the most striking cases is a wave of WGDs in different flowering plant lineages that seem to coincide with the Cretaceous/Paleogene (K/Pg) boundary (62-67). Furthermore, many of the WGDs clustered around the K/Pg extinction event are at the base of some of the largest and most successful extant plant families. Polyploidy thus somehow appears to be correlated with plant survival through the K/Pg boundary (63) and with species diversification in angiosperms (68). 
Once polyploids are formed, they must become locally established, reproduce, and survive while adapting to different environments. These processes might ultimately lead to their long-term evolutionary success, where their descendant lineages survive for tens of millions of years. Most likely, both neutral and adaptive processes contribute to polyploid establishment under stressful conditions in the short term. The adaptive scenario is mostly based on characteristics often displayed by newly formed polyploids, such as the formation of more extreme phenotypes in the resulting hybrid populations compared with their diploid parents. Moreover, genomic instability and gene expression changes soon after polyploid formation (shown to occur in both recent natural polyploids and synthetics; above) may result in increased phenotypic variability, which might be advantageous and allow rapid adaptation to changed environments and conditions (69-72). Other potential adaptive advantages of newly formed polyploids include the masking of deleterious recessive alleles leading to increased mutational robustness. The neutral scenario gets support from the fact that levels of unreduced gamete formation can be increased by external stimuli such as stress and a fluctuating environment (73). Temperature in particular has a pronounced effect on unreduced gamete formation. Moreover, increased levels of unreduced pollen in the fossil record were observed in the now-extinct conifer family Cheirolepidiaceae at the Triassic-Jurassic transition, which corresponds to the fourth of the five major extinction events (74), while abnormal gymnosperm pollen (75) and lycophyte spores (76) have also been reported from the Permian-Triassic transition, corresponding to the third of the five major extinction events. Increased unreduced gamete production during times of environmental stress and/or fluctuation could thus be an important factor in explaining the apparent clustering of paleopolyploidizations at the 
K/Pg boundary. It could also explain why many present-day polyploids often are more abundant in stressful environments, such as the Arctic (22) or disturbed habitats (77).

Although many genes undergo homeolog loss after WGD, others, particularly regulatory and developmental genes, are retained in excess after WGD. This pattern of gene loss and retention is most likely due to dosage-balance constraints and selection against loss of individual components of completely duplicated macromolecular complexes and/or pathways, because this would disrupt their overall stoichiometry (78-81). Retention of dosage-sensitive duplicates thus does not provide an immediate evolutionary advantage and adaptation, but results from the fact that their loss would lead to an immediate disadvantage. In this respect, the retained regulators may be considered an evolutionary spandrel (82), which might later on facilitate evolutionary innovations and/or diversifications. Selection to maintain dosage balance eventually relaxes over time allowing functional divergence and duplicated networks to be rewired to evolve novel functionality and increase biological complexity (83), which could help explain the vast post-WGD success observed in some of the plant families that experienced a WGD at the K/Pg boundary.

\section{Synthesis: Linking Microevolutionary Processes with Macroevolutionary Patterns}

High levels of unreduced gamete formation in natural populations of angiosperms (e.g. 19, 20) provide a mechanism for polyploid formation and ultimately, speciation, via allopolyploidy (involving interspecific hybridization) and autopolyploidy (formed within a species). Both modes of formation contribute substantially to angiosperm species diversity 
(84). Nascent polyploids undergo an array of genomic and expression-level processes that may result simply from the presence of multiple genomes within the same nucleus.

Although resolution of this multi-genome challenge may take multiple forms (chromosomal restructuring, homeolog loss, alterations in gene expression, etc.), it is clear that polyploids are not merely the additive products of their diploid progenitors. Instead, they are mosaics of parental, additive, and novel features, and even young polyploid species appear to be composed of arrays of genetically unique individuals. Moreover, diploidization processes, while returning a polyploid to a diploid-like state, do not return the polyploid to the original diploid state - that is, some loci are retained in duplicate, singletons may derive from one parent or the other, and shifts in gene expression (neo- and subfunctionalization) render the diploidized polyploid unique. This novelty and range of phenotypic diversity may provide polyploid species with unusual adaptive capacity, particularly in times of high environmental stress. In fact, WGD events in angiosperms are non-randomly associated with bursts in diversification (68), and these radiations tend to be marked by novelty in morphology and/or chemistry. It is intriguing indeed to consider that the processes we observe in recent polyploids may explain patterns of WGD and key innovations across macroevolutionary timescales.

\section{Acknowledgments}

This work was supported in part by NSF grants DEB-0919254, DEB-0922003, DEB-

1146065, and EF-1115210. 


\section{Literature Cited}

1. Dodsworth, S., A. R. Leitch, and I. J. Leitch. Genome size diversity in angiosperms and its influence on gene space. Current Opinions in Genes and Development, this issue.

2. Leitch, I.J., and A.R. Leitch. 2013. Genome size diversity and evolution in land plants. In: Leitch I.J., J. Greilhuber, J. Doležel, and J.F. Wendel [eds.], Plant Genome Diversity, Volume 2, 307-322. Springer, Vienna, Austria. This chapter is an excellent compendium of genome size variation in plants and of the mechanisms that shape plant genome sizes.

3. Stebbins Jr, G. L. (1947). Types of polyploids; their classification and significance. Advances in Genetics 1: 403-429.

4. Stebbins, G. L. 1950. Variation and evolution in plants. New York: Columbia University Press. Stebbins devotes multiple chapters to the topic of polyploidy - from the processes involved to the genetic consequences to paleopolyploidy.

5. Grant V. 1971. Plant speciation. New York, USA: Columbia University Press.

6. Grant V. 1981. Plant speciation. $2^{\text {nd }}$ ed. New York, USA: Columbia University Press. Chapters of this book focus on hybridization, polyploidy, and frequency in nature and provide both a conceptual framework and empirical examples. 
7. Soltis, D. E. and P. S. Soltis. 1993. Molecular data and the dynamic nature of polyploidy. Critical Reviews in Plant Sciences 12: 243-273.

8. Soltis, P. S. and D. E. Soltis. 2000. The role of genetic and genomic changes in the success of polyploids. Proceedings of the National Academy of Sciences, USA 97: 7051-7057. Rapid genetic and genomic changes may accompany polyploidy and could be responsible for the success of these organisms.

9. Soltis, P. S. and D. E. Soltis. 2009. The role of hybridization in plant speciation. Annual Review of Plant Biology 60: 561-588.

10. Otto, S.P., and J. Whitton. 2000. Polyploidy incidence and evolution. Annual Review of Genetics 34:401-437.

11. Masterson, J. 1994 Stomatal size in fossil plants: evidence for polyploidy in majority of angiosperms. Science 264, 421-424.

12. Muntzing A. 1936. The evolutionary significance of autopolyploidy. Hereditas 21:263378. An early paper detailing the characteristics of autopolyploids and their importance in nature. 
13. Darlington, C.D. 1937. Recent Advances in Cytology. P. Blakiston's Son \& Co., Philadelphia. Pennsylvania, USA. Put forth the idea that the degree of parental divergence drives polyploid chromosome pairing patterns (allo- vs. autopolyploidy).

14. Grant. V. 1975. Genetics of flowering plants. New York, USA: Columbia University Press.

15. Vision, T.J., D.G. Brown, and S.D. Tanksley. 2000. The origins of genomic duplications in Arabidopsis. Science 290(5499):2114-2117.

16. Bowers, J.E., B.A. Chapman, J. Rong, and A.H. Paterson. 2003. Unravelling angiosperm genome evolution by phylogenetic analysis of chromosomal duplication events. Nature 422(6930):433-438.

17. Jiao, Y., N. J.Wickett, S. Ayyampalayam, A. S. Chanderbali, L. Landherr, P. E. Ralph, L. P. Tomsho, Y. Hu, H. Liang, P. S. Soltis, D. E. Soltis, S. W. Clifton, S. E. Schlarbaum, S. C. Schuster, H. Ma, J. Leebens-Mack, and C. W. dePamphilis. 2011. Ancestral polyploidy in seed plants and angiosperms. Nature 473: 97-100.

18. Levin, D. A. 1975. Minority cytotype exclusion in local plant populations. Taxon 24: 3543.

19. Ramsey J, Schemske DW. 1998. Pathways, mechanisms, and rates of polyploid formation in flowering plants. Annu. Rev. Ecol. Evol. Syst. 29:467-501. An excellent 
review of polyploidy, especially focusing on mechanisms of formation and rates of polyploidization.

20. Ramsey J, Schemske DW. 2002. Neopolyploidy in flowering plants. Annu. Rev. Ecol. Evol. Syst. 33:589-639.

21. Ehrendorfer, F. 1980 Polyploidy: Biological Relevance (ed. Lewis, W.) New York, USA: Plenum Press.

22. Brochmann C, Brysting AK, Alsos IG, Borgen L, Grundt HH, Scheen AC, Elven R. 2004 Polyploidy in arctic plants. Biol. J. Linn. Soc. 82, 521-536.

23. Roose, M. L. \& Gottlieb, L. D. 1976 Genetic and biochemical consequences of polyploidy in Tragopogon. Evol. 30, 818-830.

24. Soltis DE, Rieseberg LH. 1986. Autopolyploidy in Tolmiea menziesii (Saxifragaceae): Genetic insights from enzyme electrophoresis. Am. J. Bot. 73:310-318.

25. Soltis, D. E. and P. S. Soltis. 1989. Genetic consequences of autopolyploidy in Tolmiea (Saxifragaceae). Evolution 43: 586-594.

26. Levin, D. A. 1983 Polyploidy and novelty in flowering plants. Am. Nat. 122, 1-25. A seminal paper that highlighted the potential advantages of autopolyploidy during a 
time in which polyploidy was generally thought to be maladaptive.

27. Soltis, P. S., X. Liu, D. B. Marchant, C. V., and D. E. Soltis 2014. Polyploidy and novelty: Gottlieb's legacy. Phil. Trans. R. Soc. B 369: 20130351.

28. Haufler, C. H. 1987. Electrophoresis is modifying our concepts of evolution in homosporous pteridophytes.American Journal of Botany 74:953-966.

29. Wendel, J. F. 2015. The wondrous cycles of polyploidy in plants. American Journal of Botany 102: 1-4.

30. Soltis DE, Visger CJ, Marchant DB, Soltis PS. 2016. Polyploidy: pitfalls and paths to a paradigm. Amer. J. Bot. submitted.

31. Leitch AR, Leitch IJ: Genomic plasticity and the diversity of polyploid plants Science 2008, 320: 481-483.

32. Schnable PS, Ware D, Fulton RS, Stein JC, Wei F, Pasternak S, Liang C, et al. 2009. The B73 maize genome: complexity, diversity, and dynamics Science , 326: 1112-1115.

33. Devos KM, Brown JKM, Bennetzen JL: Genome size reduction through illegitimate recombination counteracts genome expansion in Arabidopsis Genome Research 2002, 12: 1075-1079. 
34. Bennetzen JL, Ma J, Devos KM: Mechanisms of recent genome size variation in flowering plants Annals of Botany 2005, 95: 127-132.

35. Vitte C, Bennetzen JL: Analysis of retrotransposon structural diversity uncovers properties and propensities in angiosperm genome evolution Proceedings of the National Academy of Sciences 2006, 103: 17638-17643.

36. Nystedt B, Street NR, Wetterbom A, Zuccolo A, Lin YC, Scofield DG, Vezzi F, et al: The Norway spruce genome sequence and conifer genome evolution Nature 2013, 497: 579584.

37. Mestiri I, Chagué V, Tanguy AM, Huneau C, Huteau V, Belcram H, Coriton O, Chalhoub B, Jahier J: Newly synthesized wheat allohexaploids display progenitor-dependent meiotic stability and aneuploidy but structural genomic additivity New Phytologist 2010, 186: 86101.

38. Xiong Z, Gaeta RT, Pires JC: Homoeologous shuffling and chromosome compensation maintain genome balance in resynthesized allopolyploid Brassica napus. Proceedings of the National Academy of Sciences 2011, 108: 7908-7913.

39. Chester M, Gallagher JP, Symonds VV, da Silva AVC, Mavrodiev EV, Leitch AR, Soltis PS, Soltis DE. Extensive chromosomal variation in a recently formed natural allopolyploid 
species, Tragopogon miscellus (Asteraceae) Proceedings of the National Academy of Sciences 2012, 109: 1176-1181.

40. Zhang H, Bian Y, Gou X, Zhu B, Xu C, Qi B, Li N, et al: "Persistent whole-chromosome aneuploidy is generally associated with nascent allohexaploid wheat Proceedings of the National Academy of Sciences 2013, 110: 3447-3452.

41. Ainouche, M., H. Chelaifa, J. Ferreira, S. Bellot, A. Ainouche, A. Salmon, Polyploid Evolution in Spartina: Dealing with Highly Redundant Hybrid Genomes, In P.S. Soltis and D.E. Soltis [eds.], Polyploidy and Genome Evolution, (2012) 225-243.

42. Hegarty, M. J., R.J. Abbott, S.J. Hiscock, Allopolyploid Speciation in Action: The Origins and Evolution of Senecio cambrensis, In P.S. Soltis and D.E. Soltis [eds.], Polyploidy and Genome Evolution, (2012) 245-270.

43. Soltis, D. E., R. J. A. Buggs, W. B. Barbazuk, S. Chamala, M. Chester, J. P. Gallagher, P. S. Schnable, and P. S. Soltis. 2012. Rapid and repeated evolution in the early stages of polyploidy: genomic and cytogenetic studies of recent polyploidy in Tragopogon. In P. S. Soltis and D. E. Soltis (eds.), Polyploidy and Genome Evolution. Springer, Heidelberg.

44. Madlung, A., Tyagi, A. P., Watson, B., Jiang, H., Kagochi, T., Doerge, R. W., Martienssen, R. \& Comai, L. 2005 Genomic changes in synthetic Arabidopsis polyploids. Plant J. 41, 221230. 
45. Akama, S, R Shimizu-Inatsugi, K. K. Shimizu, and J. Sese. 2014. Genome-wide quantification of homeolog expression ratio revealed nonstochastic gene regulation in synthetic allopolyploid Arabidopsis. Nuclear Acids Research 1-15

46. Gaeta, R.T., J.C. Pires, F. Iniguez-Luy, E. Leon, T.C. Osborn, Genomic changes in resynthesized Brassica napus and their effect on gene expression and phenotype, Plant Cell, 19 (2007) 3403-3417.

47. Shen, Y., Y. Zhang, J. Zou, J. Meng, and J. Wang, 2015. Comparative proteomic study on Brassica hexaploid and its parents provides new insights into the effects of polyploidization, J. Proteomics, 112 (2015) 274-284.

48. Schnable, J. C., Springer, N. M. \& Freeling, M. 2011 Differentiation of the maize subgenomes by genome dominance and both ancient and ongoing gene loss. Proc. Natl. Acad. Sci. U. S. A. 108, 4069-4074.

49. Ownbey, M. 1950 Natural hybridization and amphiploidy in the genus Tragopogon. Am. J. Bot. 37, 487-499.

50. Buggs, R. J. A., S. Chamala, W. Wu, L. Gao, G. D. May, P. S. Schnable, D. E. Soltis, P. S. Soltis, and W. B. Barbazuk. 2010a. Characterization of duplicate gene evolution in the recent 
natural allopolyploid Tragopogon miscellus by next-generation sequencing and Sequenom iPLEX MassARRAY genotyping. Molecular Ecology 19: 132-146.

51. Buggs, R. J. A., N. M. Elliott, L. Zhang, J. Koh, L. F. Viccini, D. E. Soltis, and P. S. Soltis. 2010b. Tissue-specific silencing of homoeologs in natural populations of the recent allopolyploid Tragopogon mirus. New Phytologist 186: 175-183.

52. Buggs, R. J. A., L. Zhang, N. Miles, J. A. Tate, L. Gao, W. Wei, P. S. Schnable, W. B. Barbazuk, P. S. Soltis, and D. E. Soltis. 2011. Transcriptomic shock generates evolutionary novelty in a newly formed, natural allopolyploid plant. Current Biology 21: 551-556.

53. Buggs, R. J. A., S. Chamala, W. Wu, J. A. Tate, P. S. Schnable, D. E. Soltis, P. S. Soltis, W. B. Barbazuk. 2012a. Rapid, repeated, and clustered loss of duplicated genes in allopolyploid Tragopogon populations of independent origin. Current Biology 22: 248-252.

54. Buggs, R. J. A., S. Renny-Byfield, M. Chester, I. E. Jordon-Thaden, L. Facio Viccini, S. Chamala, A. R. Leitch, P. S. Schnable, W. B. Barbazuk, P. S. Soltis, and D. E. Soltis. 2012b. Next-generation sequencing and genome evolution in allopolyploids. American Journal of Botany 99: 372-382.

55. Mavrodiev, M. M. Chester, V. N. Suárez-Santiago, C. Visger, A. Susanna, R. Rodriguez, R. M. Baldini, P. S. Soltis and D. E. Soltis. 2015. Parentage, multiple origins, and distribution of 
the allotetraploid Tragopogon castellanus Levier s. l. (Asteraceae). New Phytologist doi: 10.1111/nph.13227.

56. Chen ZJ. 2007. Genetic and epigenetic mechanisms for gene expression and phenotypic variation inplant polyploids. Annu. Rev. Plant Biol. 58:377-406.

57. Wright, KM, JC Pires, and A Madlung. 2009. Mitotic instability in resynthesized and natural polyploids of the genus Arabidopsis (Brassicaceae). American Journal of Botany 96:1656-1664.

58. Wendel, J. F. and R. Cronn. 2003. Polyploidy and the evolutionary history of cotton. Advances In Agronomy vol 78. Academic Press, London, pp 139-186.

59. Paterson, A.H., Z. Wang, J. Li, and H. Tang. 2012. Ancient and recent polyploidy in the monocots. In P.S. Soltis, and D.E. Soltis [eds.], Polyploidy and Genome Evolution, 93-108. Springer-Verlag, Berlin, New York.

60. Vekemans D, Proost S, Vanneste K, Coenen H, Viaene T, Ruelens P, Maere S, Van de Peer Y, Geuten K. 2012 Gamma paleohexaploidy in the stem lineage of core eudicots: significance for MADS-box gene and species diversification. Mol. Biol. Evol. 29, 3793-3806

61. Jiao, Y., J. Leebens-Mack, S. Ayyampalayam, J. E. Bowers, M. R McKain, J. McNeal, M. Rolf, D. R. Ruzicka, E. Wafula, N. J. Wickett, X. Wu, Y. Zhang, J. Wang, Y. Zhang, E. Carpenter, M. K. 
Deyholos, T. M. Kutchan, A. S. Chanderbali, P. S. Soltis, D. W. Stevenson, R. McCombie, J. C. Pires, G. Ka-Shu Wong, D. E. Soltis, and C. W. dePamphilis. 2012. A genome triplication associated with early diversification of the core eudicots. Genome Biology 13: R3.

62. Fawcett, J., Maere, S., Van de Peer, Y. (2009) Plants with double genomes might have had a better chance to survive the Cretaceous-Tertiary extinction event. Proc Natl Acad Sci $106,5737-42$.

63. Vanneste, K., Baele, G., Maere, S., Van de Peer, Y. (2014a) Analysis of 41 plant genomes supports a wave of successful genome duplications at the Cretaceous-Tertiary boundary.

Genome Res. 24,1334-1347. An innovative analysis demonstrating widespread polyploidy at the K-T boundary, with possible explanations for the success of polyploids at times of environmental stress.

64. Vanneste, K., Maere, S., Van de Peer, Y. (2014b) Tangled up in two: A burst of genome duplications at the end of the Cretaceous and the consequences for plant evolution. Philosophical Transactions B 369, 20130353 .

65. Vanneste, K., Sterck, L., Myburg, A.A., * Van de Peer, Y., * Mizrachi, E. (2015) Horsetails are ancient polyploids: Evidence from Equisetum giganteum. The Plant Cell 27: 1567-1578

66. Cai, H., Liu, X., Vanneste, K., Proost, S., Tsai, C., Liu, K.-W., Chen, L.-J., He, Y., Xu, SX., Bian, C., Zheng, Z., Sun, F., Liu, W., , ., Pan, Z.-Y., Hsu, C.-C., Yang, Y.-P., Hsu, Y.-C., Chuang, Y.-C., 
Dievart, A., Dufayard, J.-F., Xu, SX., Wang, X., Wang, X., Xiao, X.-J., Zhao, X.-M., Du, Y., Zhang, G.-Q., Wang, X., Su, Y.-Y., Xie, G.-C., Liu, G.-H., Li, L.-Q., Huang, L.-Q., Luo, Y., Chen, H.-H., Van de Peer, Y., Liu, Z.-J. (2014) The genome sequence of the orchid Phalaenopsis equestris. Nature Genetics 47, 65-72.

67. Olsen JL, Rouzé P, Verhelst B, Lin YC, Bayer T, Collen J, Dattolo E, De Paoli E, Dittami S, Maumus F, Michel F, Kersting A, Lauritano C, Lohaus R, Topel M, Tonon T, Vanneste K, Amirebrahimi M, Brakel J, Boström C, Chovatia M, Grimwood J, Jenkins JW, Jüterbock A, Mraz A, Stam WT, Tice H, Bornberg-Bauer E, Green PJ, Pearson GA, Procaccini G, Duarte CM, Schmutz J, Reusch TBH, Van de Peer Y. 2016. Genome of the seagrass Zostera marina which has secondarily returned to the sea. Nature, in press.

68. Tank, D. C., J. M. Eastman, M. W. Pennell, P. S. Soltis, D. E. Soltis, C E. Hinchliff, J. W. Brown, and L. J. Harmon. 2015. Progressive radiations and the pulse of angiosperm diversification. New Phytologist 207: 454-467.

69.. Rieseberg LH et al. 2003 Major ecological transitions in wild sunflowers facilitated by hybridization. Science 301, 1211-1216.

70. Comai L. 2005 The advantages and disadvantages of being polyploid. Nat. Rev. Genet. 6, 836-846.

71. Madlung A. 2013 Polyploidy and its effect on evolutionary success: old questions 
revisited with new tools. Heredity 110, 99-104.

72. te Beest M, Le Roux JJ, Richardson DM, Brysting AK, Suda J, Kubesova M, Pysek P. 2012 The more the better? The role of polyploidy in facilitating plant invasions. Ann. Bot. 109, $19-45$.

73. De Storme N, Geelen D. 2013 The impact of environmental stress on male reproductive development in plants: biological processes and molecular mechanisms. Plant Cell Environ. $37,1-18$.

74. Kürschner WM, Batenburg S and Mander L (2013). Aberrant Classopollis pollen reveals evidence for unreduced (2n) pollen in the conifer family Cheirolepidiaceae during the Triassic-Jurassic transition. Proceedings of the Royal Society of London. Biological

Sciences 280 (1768:20131708). doi: 10.1098/rspb.2013.1708

75. Foster CB and Afonin SA. 2005 Abnormal pollen grains: an outcome of deteriorating atmospheric conditions around the Permian-Triassic boundary. J. Geol. Soc. 162, 653-659.

76. Visscher H, Looy CV, Collinson ME, Brinkhuis H, van Konijnenburg-van Cittert JH, Kurschner WM, Sephton MA. 2004 Environmental mutagenesis during the end-Permian ecological crisis. Proc. Natl Acad. Sci. USA 101, 12 952-12 956. 
77. Mraz P, Spaniel S, Keller A, Bowmann G, Farkas A, Singliarova B, Rohr RP, Broennimann 0, Muller-Scharer H. 2012 Anthropogenic disturbance as a driver of microspatial and microhabitat segregation of cytotypes of Centaurea stoebe and cytotype interactions in secondary contact zones. Ann. Bot. 110, 615-627.

78. Maere S, De Bodt S, Raes J, Casneuf T, Van Montagu M, Kuiper M, Van de Peer Y. 2005 Modeling gene and genome duplications in eukaryotes. Proc. Natl Acad. Sci. USA 102, 54545459

79. Birchler JA, Veitia RA. 2007. The gene balance hypothesis: from classical genetics to modern genomics. Plant Cell 19: 395-402

80. Birchler JA \& Veitia RA. 2012. Gene balance hypothesis: connecting issues of dosage sensitivity across biological disciplines. Proc Natl Acad Sci U S A 109(37):14746-14753.

81. Conant, G. C., J. A. Birchler, and J. C. Pires. 2014. Dosage, duplication, and diploidization: clarifying the interplay of multiple models for duplicate gene evolution over time. Current Opinion in Plant Biology 19: 91-98.

82. Freeling M, Thomas BC. 2006 Gene-balanced duplications, like tetraploidy, provide predictable drive to increase morphological complexity. Genome Res. 16, 805-814.

83. De Smet R, and Van de Peer Y. 2012 Redundancy and rewiring of genetic networks 
following genome wide duplication events. Curr. Opin. Plant Biol. 15, 168-176. This paper discusses the influence of polyploidy on the evolution of gene regulation and function from the perspective of gene networks rather than single genes.

84. Barker MS, Arrigo N, Baniaga AE, Li Z, Levin DA 2015. On the relative abundance of autopolyploids and allopolyploids. New Phytologist doi:10.1111/nph.13698 
Box. Glossary of terms.

Allopolyploidy - polyploidy formed through the combined processes of interspecific hybridization and genome doubling.

Autopolyploidy - genome doubling that arises within a species; it may involve a single individual or crossing between individuals from genetically distinct lineages within the species.

Diploidization - the processes that return a polyploid genome to a diploid-like genome; these may include loss of duplicate genes and chromosomes, loss of repetitive DNA, gene silencing, altered chromosome pairing.

Diploidy - the state of being diploid; that is, containing two complete sets of chromosomes (or genomes).

Fractionation - the loss of one copy of a gene pair duplicated by polyploidy; losses may be random with respect to the parental genome or biased, with most/all losses from a single parental genome.

Homeolog (also homoeolog) - chromosomes (and the genes they carry) that are duplicated by polyploidy. 
Polyploidy - the state of having more than two complete sets of chromosomes.

Polyploidization - the process(es) of polyploid formation; this can be duplication, triplication, or higher-order multiplication of a genome.

Whole-genome duplication - the duplication of a complete genome, for example, of a diploid genome (with two copies of each chromosome) to form a tetraploid (with four copies of each chromosome); this term is sometimes used to refer to the process of duplication (i.e., polyploidization) and sometimes in reference to the state of having multiple, duplicate genomes (i.e., polyploidy). 
Fig. 1. Timeline showing origin of polyploid species, generation of novelty, environmental filtering, rapid radiation from selected line, and resulting signature of paleopolyploidy. Note that diploidization processes (not pictured) may occur continuously through this timeline. A. Polyploid formation (in yellow circle) and attendant processes that yield variation in, for example, inflorescence morphology, karyotype, and homeolog loss; other novelties, e.g., altered gene expression, transposon activity, are not pictured. B. Array of polyploid genotypes/phenotypes, represented by different colors of rings around the photograph. C. A single remaining polyploid 'type' after environmental filtering or drift. D. Rapid radiation from this 'successful' polyploid and resultant formation of multiple lineages that trace back to this single common ancestor. E. Deep phylogeny showing the correspondence (turquoise ring) between ancient WGD and radiation, as derived in D. Other paleopolyploid events are also shown. Credits: J. Tate, V. Symonds, R. Buggs, M. Chester, I. Jordon-Thaden, D. Tank et al. (E modified from Tank et al. 2015). 
A Polyploid formation
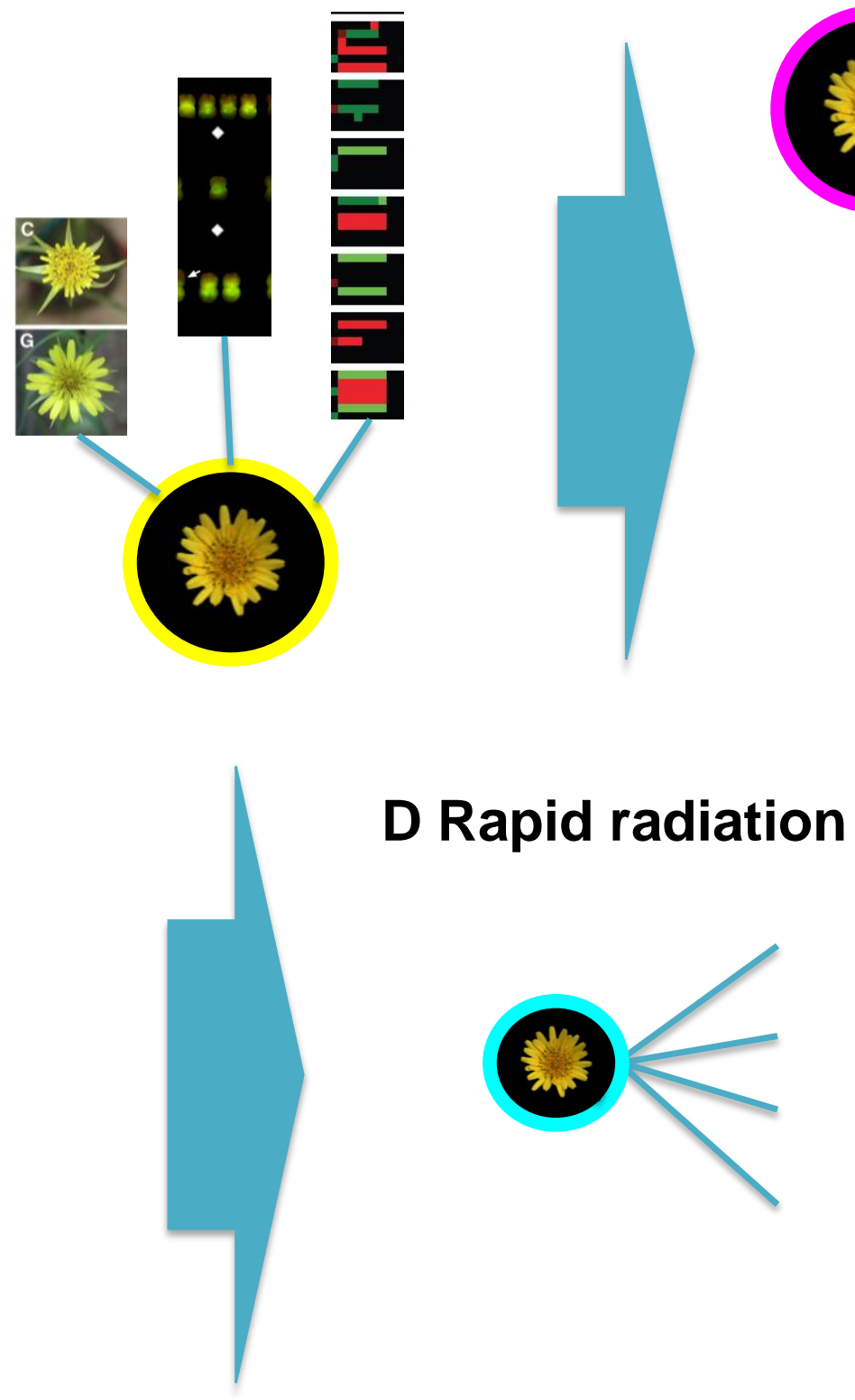

B Polyploid diversity

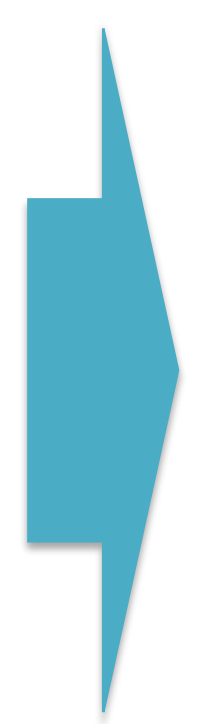

C Environmental filtering/drift
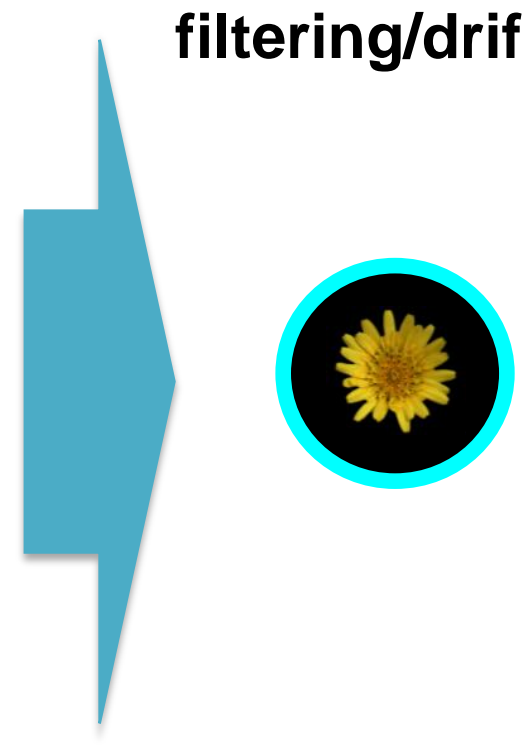

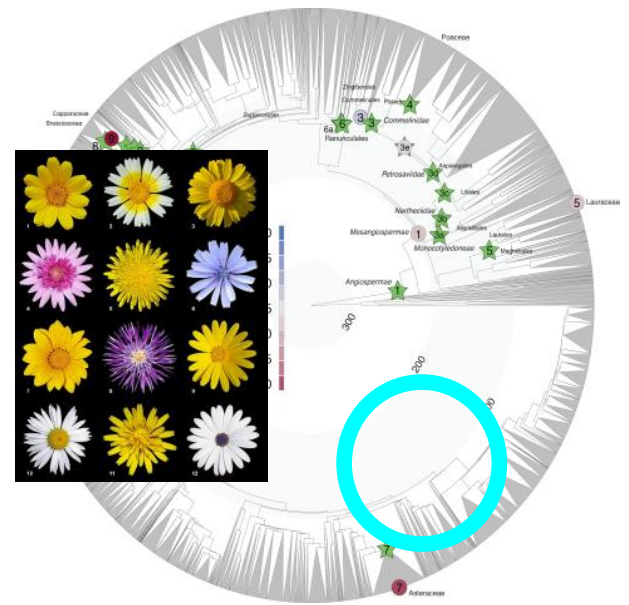

E Deep phylogeny
$\&$ ancient radiations

Deep phylogeny
\& ancient radiations

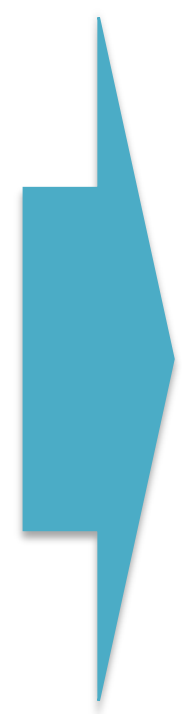

